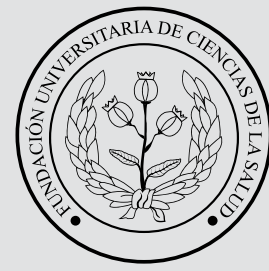

FUCS
Re

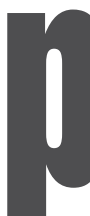

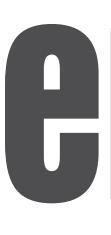

\title{
Esquemas completos aplicados contra COVID-19 priorizados por entidad territorial en Colombia
}

Completed COVID-19 vaccination among the prioritized groups by territorial entitiy in Colombia

${ }^{a}$ Ingeniero. Magister en Gestión de la Tecnologia Educativa, Especialista en Administración de la Informática Educativa. Docente de matemáticas e Investigador, Secretaría de Educación de Soacha, Cundinamarca.

\section{R ES U M EN}

Introducción: el 6 de marzo 2020 Colombia registró el primer caso de infección por SARS-CoV-2. Con la cuarentena se enfrentaba también el temor popular acerca del riesgo de seguir con la vacunación usual en tiempos de COVID-19 y de cómo esta podría influir en forma negativa en el término de la infección por SARS-CoV-2. Objetivo: mostrar el avance de la población priorizada para la vacunación contra SARS-CoV-2 en las entidades territoriales de Colombia, además de los esquemas completos y el porcentaje priorizado, en el periodo comprendido entre el 17 de febrero a 26 de julio 2021. Metodología: esta investigación es un estudio transversal, como fuente de información se obtuvo del plan nacional de vacunación contra el COVID-19 de la página web del Ministerio de Salud y Protección Social. Resultados: las entidades territoriales con mayor número de población priorizada para la vacunación fueron: Antioquia (2'583.113), Bogotá (2'077.324), Valle del Cauca (1'437.805), Cundinamarca (1'095.364) y Santander (797.740); entre las entidades territoriales con mayor número de esquemas completos de vacunación estuvieron: Bogotá (1'168.212), Antioquia (620.133), Valle del Cauca (576.283), Santander (335.015) y Norte de Santander (232.110). Se concluyó que los territorios con mayor número de porcentaje priorizado de vacunación fueron: Amazonas (128\%), Vaupés (119,92\%), Guainía (93,6\%), Bogotá (56,24\%) y Quindío (51, 73\%). Conclusión: es fundamental seguir los resultados de los esquemas de vacunación a nivel territorial para entender su eficacia, la posible reducción de la respuesta inmune con el tiempo y las posibles consecuencias adversas.

Palabras clave: COVID-19, SARS-CoV-2, pandemia, vacunas.

(C) 2021 Fundación Universitaria de Ciencias de la Salud - FUCS Este es un artículo Open Access bajo la licencia CC BY-NC-ND (http://creativecommons.org/licenses/by-nc-nd/4.0/).

INFORMACIÓN DEL ARTÍCULO

Historia del artículo:

Fecha recibido: agosto 3 de 2021 Fecha aceptado: septiembre 1 de 2021
Autor para correspondencia: Ing. Jorge Enrique Díaz Pinzón jediazp@unal.edu.co
DOI

10.31260/RepertMedCir.01217372.1259 
Introduction: Colombia registered the first case of SARS-CoV-2 infection on March 6 2020. A general feeling of fear on the risk of continuing usual vaccination programs in times of COVID-19 and how this could negatively impact on the SARSCoV-2 infection term, was also faced during the quarantine. Objective: to measure progress withSARS-CoV-2 vaccination among prioritized populations, as well as the proportion of completed vaccination and vaccination percentage of this target population, by territorial entities in Colombia, in the period from February 17 to July 26 2021. Methodology: a cross-sectional study research. Data was obtained from the Ministry of Health and Social Protection website based on the national COVID-19 vaccination plan. Results: the territorial entities with the highest population in the priority vaccination groups were: Antioquia (2'583.113), Bogota (2'077.324), Valle del Cauca (1'437.805), Cundinamarca (1'095.364) and Santander (797.740); among the territorial entities with the highest proportion of people who have received completed vaccination schedules were: Bogota (1'168.212), Antioquia (620.133), Valle del Cauca (576.283), Santander (335.015) and Norte de Santander (232.110). It was concluded that the territories with the highest vaccination percentage in the prioritized groups were: Amazonas $(128 \%)$, Vaupes $(119.92 \%)$, Guainia $(93,6 \%)$, Bogota $(56,24 \%)$ and Quindio $(51,73 \%)$. Conclusion: monitoring the results of completed vaccination at the territorial level is essential to gain understanding on the effectiveness, a possible drop of immune response over time, and the potential adverse consequences of these vaccines.

Key words: COVID-19, SARS-CoV-2, pandemic, vaccines.

(C) 2021 Fundación Universitaria de Ciencias de la Salud - FUCS. This is an open access article under the CC BY-NC-ND license (http://creativecommons.org/licenses/by-nc-nd/4.0/).

\section{INTRODUCCIÓN}

El 6 de marzo 2020 Colombia registró el primer caso de infección por SARS-Cov-2. ${ }^{1}$ Con la cuarentena también el temor popular acerca del riesgo de seguir con la vacunación usual en tiempos de COVID-19 y de cómo esta podría influir negativamente el término de la infección por SARS-CoV-2, sobre todo en personas que fueran vacunadas contra otros patógenos como la influenza, a pesar de que esta interacción acreditada como interferencia viral, ya ha sido descrita y descartada con otros virus similares. ${ }^{2-4}$

Esta epidemia no es un buen instante para enfermar ni para solicitar atención médica, ya que todos los esfuerzos del sistema de salud están encaminados a enfrentar el SARS-CoV-2, de manera que tener una gran parte de la población vacunada, impedirá una significativa morbilidad que sature aún más el sistema de atención en salud. ${ }^{5}$

Para conseguir una reducción continuada de los casos de infección ${ }^{6}$ se necesitan contramedidas múltiples incluidos el distanciamiento, las pruebas y el rastreo. La reciente aparición de nuevas variantes del SARS-CoV- $2^{7}$ como B.1.1.7 y B.1.351, según se informa tienen una mayor transmisibilidad $^{8,9}$ y lo más probable es que causarán una enfermedad más grave ${ }^{10}$ en comparación con la cepa original. No se espera que la vacunación por sí sola pueda contrarrestar la propagación de la infección, por lo tanto es necesario regular una campaña de vacunación planificada. ${ }^{11,12}$
El objetivo de esta investigación es mostrar el avance de la población priorizada para la vacunación contra SARSCoV-2 en las entidades territoriales de Colombia, además de los esquemas completos y el porcentaje priorizado, entre el periodo comprendido entre el 17 de febrero y 26 de julio 2021.

\section{METOD OLOGÍA}

La presente investigación se realizó bajo un estudio transversal, como fuente de información se obtuvo del plan nacional de vacunación contra el COVID-19 de la página web del Ministerio de Salud y Protección Social ${ }^{13}$, en el periodo comprendido entre el 17 de febrero 2021 y el 26 de julio 2021.

\section{RESULTADOS}

En la figura 1 podemos apreciar los valores de la población priorizada para la vacunación contra SARS-CoV-2 en las entidades territoriales de Colombia al 26 de julio 2021. Se puede inferir que las entidades territoriales con mayor número de población priorizada para la vacunación son: Antioquia (2'583.113), Bogotá (2'077.324), Valle del Cauca (1'437.805), Cundinamarca ( $\left.1^{\prime} 095.364\right)$ y Santander (797.740) y las de menor número son: Vaupés (4.623), Guainía (8.590), Vichada (18.624), Amazonas (18.682) y San Andrés y Providencia (36.738). 


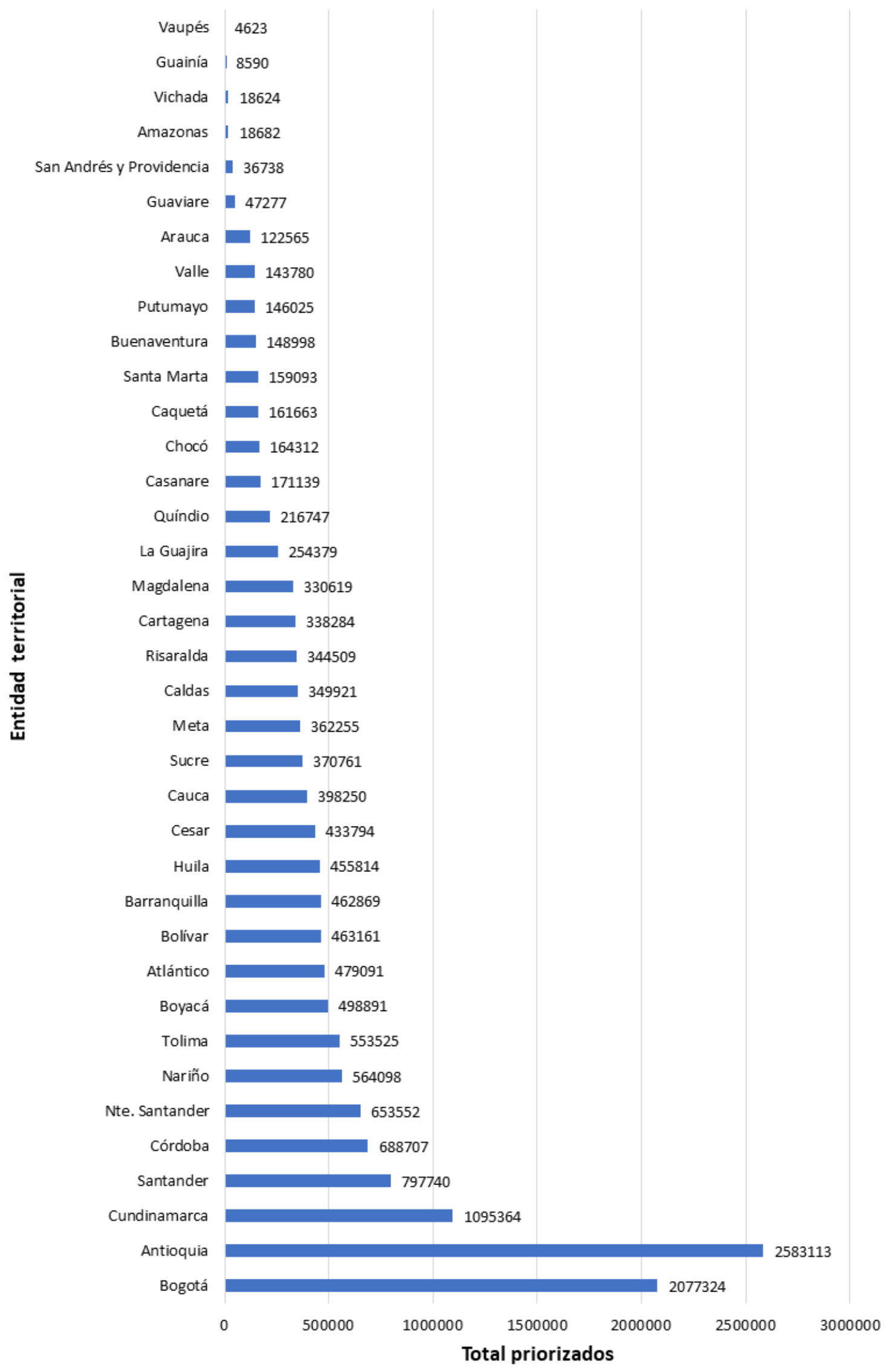

Figura 1. Población priorizada para la vacunación por entidad territorial. Fuente: el autor. 


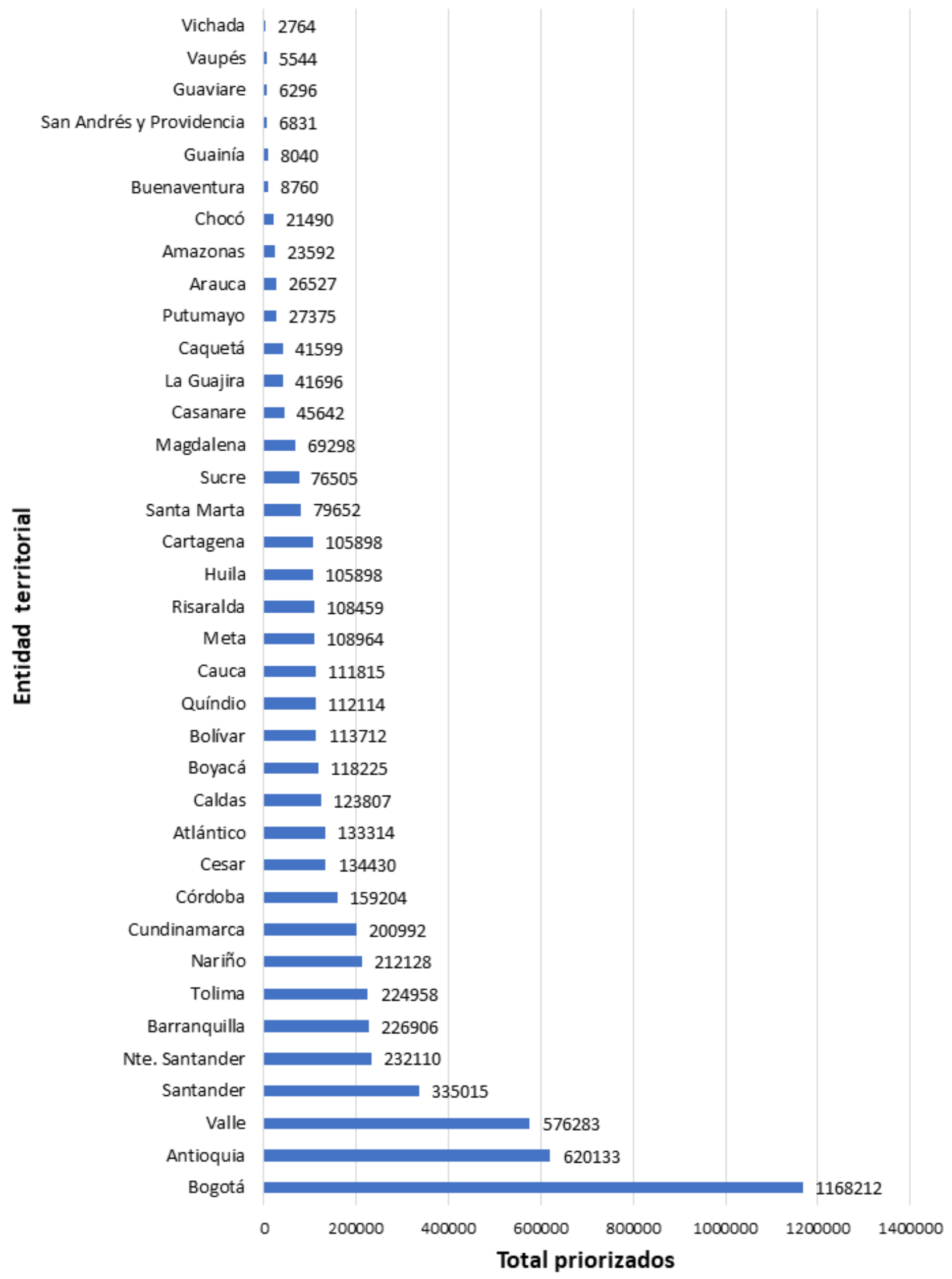

Figura 2. Esquemas completos de vacunación por entidad territorial. Fuente: el autor. 


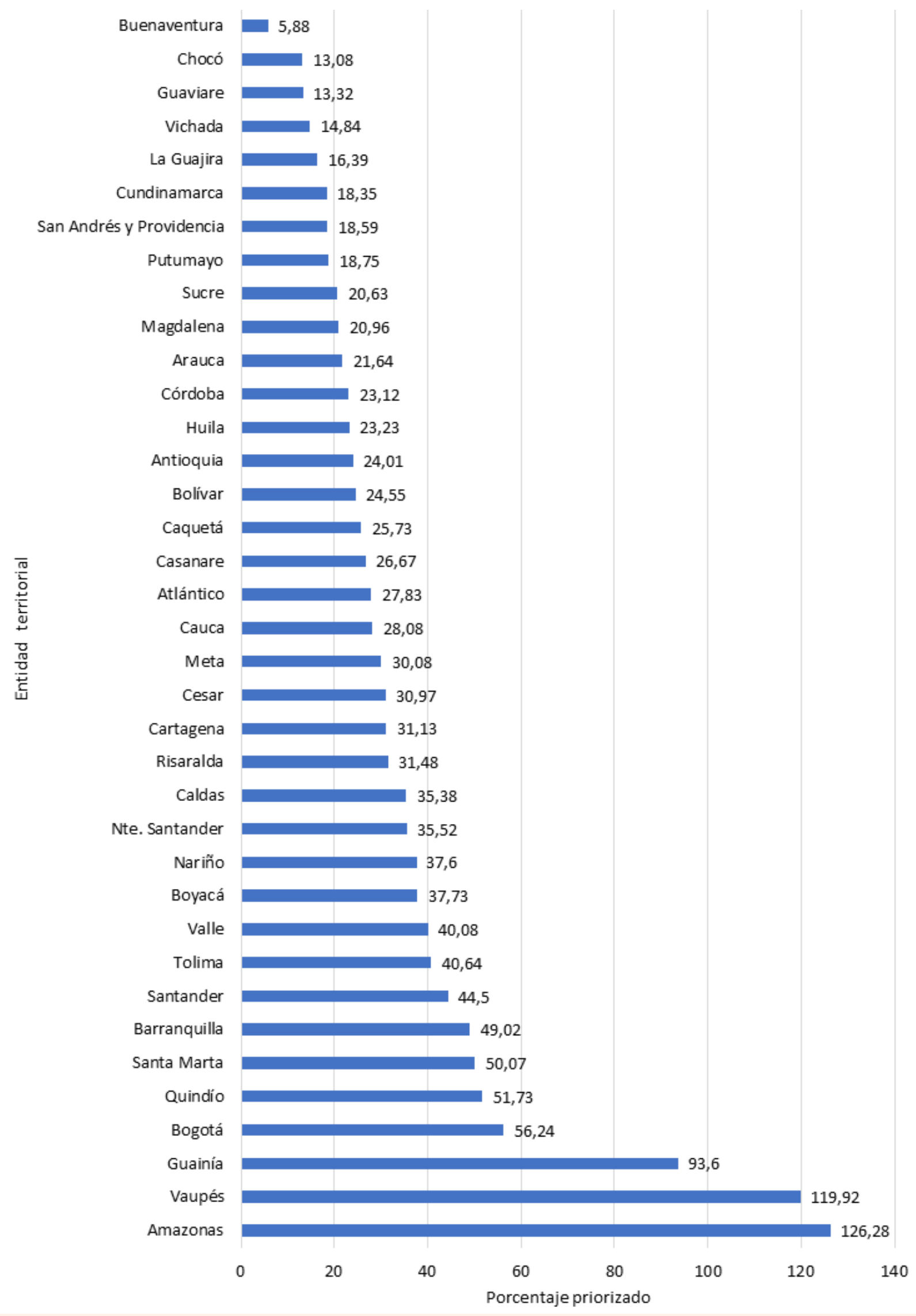

Figura 3. Porcentaje priorizado de vacunación por entidad territorial. Fuente: el autor. 
En la figura 2 podemos considerar los valores de los esquemas completos para la vacunación contra SARSCoV-2 en las entidades territoriales de Colombia, al 26 de julio de 2021. Obsérvese que aquellas con mayor número de esquemas completos de vacunación son: Bogotá (1'168.212), Antioquia (620.133), Valle del Cauca (576.283) Santander (335.015) y Norte de Santander (232.110), y con menor número son: Vichada (2.764), Vaupés (5.544), Guaviare (6.296), San Andrés y Providencia (6.831), y Guainía (8.040).

En la figura 3 podemos observar los valores del porcentaje priorizado para vacunación contra SARS-CoV-2 en las entidades territoriales de Colombia, al 26 de julio de 2021. Nótese que las entidades territoriales con mayor porcentaje de vacunación son: Amazonas (126,28\%), Vaupés (119,92\%), Guainía $(93,6 \%)$, Bogotá $(56,24 \%)$ y Quindío $(51,73 \%)$, y las de menor número son: Buenaventura $(5,88 \%)$, Chocó $(13,08 \%)$, Guaviare $(13,32 \%)$, Vichada $(14,84 \%)$ y La Guajira $(16,39 \%)$.

\section{CONCLUSIONES}

Se determinó que al 26 de julio de 2021 las entidades territoriales con mayor número de población priorizada para la vacunación fueron: Antioquia $\left(2^{\prime} 583.113\right)$, Bogotá (2'077.324), Valle del Cauca (1'437.805), Cundinamarca ( $\left.1^{\prime} 095.364\right), y$ Santander (797.740), las entidades territoriales con mayor número de esquemas completos de vacunación fueron: Bogotá (1'168.212), Antioquia (620.133), Valle del Cauca (576.283), Santander (335.015), y Norte de Santander (232.110), y se concluyó que las entidades territoriales con mayor número de porcentaje priorizado de vacunación fueron: Amazonas (128\%), Vaupés (119,92\%), Guainía $(93,6 \%)$, Bogotá $(56,24 \%)$ y Quindío $(51,73 \%)$.

Según Díaz ${ }^{14}$ a partir de la vacunación contra COVID-19 a mediados de febrero de 2021 en Colombia a mayo de 2021 no se había evidenciado un registro de la disminución del número de contagios. Este estudio aporta los primeros datos del país sobre el impacto de la vacuna contra el SARS-CoV-2 en la población.

Es fundamental seguir los resultados de los esquemas de vacunación a nivel territorial para entender su eficacia y la posible reducción de la respuesta inmune con el tiempo y las consecuencias adversas. Al 26 de julio 2021 se han aplicado en Colombia 25'641.086 vacunas y un total de esquemas completos de 11'457.631.

Hay que estar alertas a las mutaciones del virus, que pueden afectar la inmunidad proporcionada por las vacunas. Es necesario realizar un seguimiento continuo del impacto de estos cambios en la eficacia de las vacunas existentes y ejecutar si es preciso ajustes al plan de vacunación nacional.

\section{CONFLICTOS DE INTERESES}

El autor declara no tener ningún tipo de conflicto de interés.

\section{REFERENCIAS}

1. Ministerio de Salud y Protección Social de Colombia. Boletín de Prensa No 050 de 2020: Colombia confirma su primer caso de COVID-19 [Internet]. Ministerio de Salud y Protección Social de Colombia; Colombia. 2020 [Consultado 3 de mayo 2021]. Disponible en: https://www.minsalud.gov.co/Paginas/Colombiaconfirma-su-primer-caso-de-COVID-19.aspx

2. Wolff GG. Influenza vaccination and respiratory virus interference among Department of Defense personnel during the 2017-2018 influenza season. Vaccine. 2020;38(2):350-354. doi: 10.1016/j. vaccine.2019.10.005

3. Díaz Pinzón JE. Estimación de la prevalencia del COVID-19 en Colombia. Repert Med Cir. 2020;29(Núm. Supl.1):99-102. https:// doi.org/10.31260/RepertMedCir.01217372.1115

4. Díaz Pinzón JE. Análisis de los resultados del contagio del COVID-19 respecto a su distribución geográfica en Colombia. Repert Med Cir. 2020;29(Núm. Supl.1):60-64. https://doi.org/10.31260/ RepertMedCir.01217372.1082

5. Cataño JC, Aguirre HD. Vacunación del adulto en tiempos de COVID-19. Corporación de Investigaciones Biológicas. 2020;1(2):19.

6. Priesemann V, Brinkmann MM, Ciesek S, Cuschieri S, Czypionka T, et al. Calling for pan-European commitment for rapid and sustained reduction in SARS-CoV-2 infections. Lancet. 2021;397(10269):9293. doi: 10.1016/S0140-6736(20)32625-8

7. Priesemann V, Balling R, Brinkmann MM, Ciesek S, Czypionka T, et al. An action plan for pan-European defence against new SARSCoV-2 variants. Lancet. 2021;397(10273):469-470. doi: 10.1016/ S0140-6736(21)00150-1

8. Davies NG, Abbott S, Barnard RC, Jarvis CI Kucharski AJ, Munday JD, et al. Estimated transmissibility and impact of SARS-CoV-2 lineage B.1.1.7 in England. Science. 2021;372(6538):eabg3055. doi: 10.1126/science.abg 3055

9. Abbott S, Funk S, CMMID COVID-19 Working Group. Local area reproduction numbers and S-gene target failure [Internet]. Centre for Mathematical Modelling of Infectious Diseases; 2021 [citado 14 mayo 2021]. Disponible: https://cmmid.github.io/topics/covid19/ local-r-sgtf.html

10. Horby P, Huntley C, Davies N, Edmunds J, et al. NERVTAG paper on COVID-19 variant of concern B.1.1.7 [Internet]. Department of Health and Social Care and Scientific Advisory Group for Emergencies; 2021 [citado 14 mayo 2021]. Disponible en: https:// www.gov.uk/government/publications/nervtag-paper-on-covid19-variant-of-concern-bl17. 
11. Bubar KM, Reinholt K, Kissler SM, Lipsitch M, Cobey S, et al. Model-informed COVID-19 vaccine prioritization strategies by age and serostatus. Science. 2021;371(6532):916-921. doi: 10.1126/ science.abe6959

12. Ramos AM, Vela-Pérez M, Ferrández MR, Kubik AB, Ivorra, B. Modeling the impact of SARS-CoV-2 variants and vaccines on the spread of COVID-19. Commun Nonlinear Sci Numer Simul. 2021;102:105937. doi: 10.1016/j.cnsns.2021.105937
13. Ministerio de Salud y Protección Social de Colombia. Plan de vacunación nacional contra COVID-19 [Internet]. Ministerio de Salud y Protección Social de Colombia; Colombia. 2021 [Consultado 3 de mayo 2021]. Disponible en: https://www.minsalud.gov.co/ salud/publica/Vacunacion/Paginas/Vacunacion-covid-19.aspx

14. Díaz Pinzón JE. Dinámica y relación del contagio del COVID-19 después de iniciado el plan de vacunación contra el SARS-COV-2 en Colombia. Repert Med Cir. 2021;30(Núm. Supl.1):41-45. https://doi.org/10.31260/RepertMedCir.01217372.1227 